\title{
Migração: saúde reprodutiva e estereótipo
}

\section{Human migration: reproductive health and stereotypes}

\author{
Maria Silvia de Morais ${ }^{1}$ (D), Beatriz Padilla ${ }^{2,3}$ (D), Camila Morita Rosseto 4 (D), \\ Margareth Aparecida Santini de Almeida ${ }^{5}$ (D) \\ 'Departamento de Epidemiologia e Saúde Coletiva, Faculdade de Medicina de São José do Rio Preto (FAMERP) - São José do Rio \\ Preto (SP), Brasil. \\ ${ }^{2}$ University of South Florida - Tampa (FL), United States \\ ${ }^{3}$ Instituto Universitário de Lisboa (ISCTE-IUL) - Lisboa, Lisboa. \\ ${ }^{4}$ Faculdade de Medicina de São José do Rio Preto (FAMERP) - São José do Rio Preto (SP), Brasil. \\ ${ }^{5}$ Departamento de Saúde Pública, Faculdade de Medicina de Botucatu, Universidade Estadual Paulista (UNESP) - Botucatu (SP), \\ Brasil.
}

Como citar: Morais MS, Padilla B, Rosseto CM, Almeida MAS. Migração: saúde reprodutiva e estereótipo. Cad Saúde Colet, 2021;29(esp.):86-91. https://doi.org/10.1590/1414-462X202199010337

\section{Resumo}

Introdução: A saúde é um direito universal em Portugal garantido pela Lei n 56/79, artigo 4º, mas fatores sociais de gênero e de nacionalidade têm condicionado o seu acesso, a exemplo do preconceito sofrido pelas brasileiras residentes nesse país e da falta de acesso em relação à saúde reprodutiva vivenciados por elas. Objetivo: Analisar a percepção de mulheres imigrantes brasileiras em relação aos serviços de saúde reprodutiva em Portugal. Método: Foram realizadas entrevistas com 13 mulheres selecionadas mediante a técnica de amostragem em bola de neve. Resultados: As brasileiras revelaram preferência pela cesariana, o que pode colaborar para a existência de preconceito nos serviços públicos (Serviço Nacional de Saúde), alegando que eles fazem apenas parto normal sem o uso de analgesia, além de ser conduzido por enfermeiros. Nos atendimentos particulares, a cesariana normalmente é realizada, e não foi notada a presença de preconceito. Conclusão: Tal predileção, provavelmente, não esteja apenas relacionada às dificuldades experimentadas pelas imigrantes no Serviço Nacional de Saúde de Portugal, mas também à cultura favorável à cesariana, sobretudo no estado de São Paulo, que as brasileiras levam consigo ao migrarem, seja por questões de saúde ou de estética Palavras-chave: migração; imigração; emigração; saúde reprodutiva; preconceito.

\begin{abstract}
Background: In Portugal, health is a legal universal right (Law no. 56/79, art. 4); however, people's access to it is conditioned by social factors such as gender and nationality, and the case of prejudice against Brazilian women who live in that country, especially regarding their reproductive health, is an example of this conditioning. Objective: Analyze the immigrating Brazilian women's perception of the reproductive health services in Portugal. Method: Interviews were conducted with 13 women selected using the snowball sampling method. Results: The Brazilian women showed a preference for Cesarean section, which may contribute to increasing the prejudice against them in the Portuguese public health system (SNS). They argued that, in the SNS, normal delivery is performed by nurses without the use of anesthetics. Cesarean sections are frequently carried out in the private health system, where the women surveyed did not notice the presence of prejudice. Conclusion: This predilection probably is not only related to the difficulties immigrants experience in the SNS, but also to the culture favoring Cesarean section that exists, especially in the state of São Paulo, Brazil, which is carried with these women when they emigrate, due to either health or esthetic issues.
\end{abstract}

Keywords: migration; immigration; emigration; reproductive health; prejudice.

Trabalho realizado na Faculdade de Medicina de São José do Rio Preto (FAMERP) - São José do Rio Preto (SP), Brasil. Correspondência: Maria Silvia de Morais. E-mail: msmoraes@famerp.br

Este é um artigo publicado em acesso aberto (Open Access) sob a licença Creative Commons Attribution, que permite uso, distribuição e reprodução em qualquer meio, sem restrições desde que o trabalho original seja corretamente citado. 


\section{INTRODUÇÃO}

O deslocamento humano é um importante fator de encontro entre atores sociais com diferentes histórias e valores ${ }^{1}$, que compõem um mosaico de interesses. Nesse contexto, conflitos tendem a aparecer e preconceitos tendem a ser expostos, o que reflete tanto no processo de viver e adoecer como no acesso aos serviços de saúde.

A migração, em si, não constitui uma ameaça à saúde das pessoas, mas, dependendo das condições em que é realizada, pode haver uma maior ou menor vulnerabilidade. Fatores culturais, estereótipos de gênero, hábitos tradicionais das sociedades, linguagem e padrões de comunicação, entre outros, agravam as vulnerabilidades durante os processos de migração, uma vez que diferem entre culturas e, muitas vezes, influenciam o acolhimento das pessoas dentro dos países.

Esse cenário é ainda pior quando imigrantes vivenciam uma contínua exclusão social que constitui uma barreira ao acesso à saúde ${ }^{2,3}$. Além disso, quando conseguem acesso aos serviços, essas pessoas, muitas vezes, acabam sofrendo com estereótipos e não são tratadas de forma igualitária ${ }^{4,5}$

Nesse sentido, os estereótipos, ao serem "[...] representações cristalizadas sobre um grupo social, como imagens fictícias ou esquemas culturais preexistentes que expressam um imaginário social" "6:3, acabam tendo um caráter negativo na relação estabelecida entre os sujeitos, produzindo uma visão deformada que pode levar a preconceitos.

Um exemplo disso é o tratamento que as mulheres brasileiras recebem em Portugal, visto que elas têm uma imagem muito associada à sensualidade, à exotização ${ }^{4}$, levando-as a vivenciar preconceitos por parte dos profissionais, ao considerá-las como muito liberais ou as associarem à prostituição ${ }^{7}$. Consequentemente, essas mulheres imigrantes podem ter prejuízos em termos de cuidado de saúde.

Ao longo dos anos, a migração brasileira em Portugal tem atravessado várias fases da história. Nas décadas de 1980 e 1990, os imigrantes eram, em sua maioria, profissionais liberais (dentistas, publicitários e engenheiros); já a partir do ano 2000, a migração de trabalhadores para nichos menos qualificados no mercado de trabalho passou a ser majoritária. Entre os anos de 2010 a 2015, houve um recuo da migração brasileira em Portugal, que foi retomada em 2016. Estudos indicam características diferenciadas desse processo migratório e uma crescente feminização do processo, ou seja, cada vez mais mulheres estão migrando8.

Dos imigrantes brasileiros que residem em Portugal, 84\% têm entre 20 e 44 anos; $57 \%$ são do sexo feminino; a maioria é oriunda dos estados de Minas Gerais, São Paulo e Paraná; e $50 \%$ moram na área metropolitana de Lisboa ${ }^{9}$.

Portugal se insere no modelo de migração do sul da Europa caracterizado, apesar de ter algumas particularidades, pela crescente feminização dos fluxos migratórios, por possuir um mercado de trabalho segmentado por questões étnicas, etárias, de gênero e relacionadas com a escolaridade ${ }^{10}$.

Apesar de a saúde ser uma característica fundamental a ser levada em conta no processo migratório, muitos estudos de deslocamentos populacionais focalizam apenas o trabalho e a economia desses processos. Deste modo, são desconsideradas as questões em saúde e a complexidade da vida dos migrantes ${ }^{5}$.

Quando é feito o recorte para a população feminina e são analisadas as questões de saúde reprodutiva, o quadro ainda é mais preocupante. Isso se dá porque não só a situação de imigrante das mulheres faz diferença, mas também estereótipos de gênero e moralidade dentro da cultura influenciam a maneira como essas pacientes são atendidas nos serviços de saúde. Desse modo, investigações apontam para uma maior taxa de morbimortalidade materna entre as imigrantes, uma menor adesão às orientações de prevenção e um menor índice de utilização dos serviços de saúde, quando comparado com os de mulheres portuguesas ${ }^{3}$.

Tanto em Portugal como no Brasil, a saúde constitui um campo de direito universal, contudo, em Portugal, o cidadão precisa pagar taxas moderadoras de acordo com o serviço de que fizer uso. Nesse país, o acesso se dá pelo Serviço Nacional de Saúde (SNS), e, no Brasil, por meio do Sistema Único de Saúde (SUS). Nos dois países, são previstos também os serviços 
da iniciativa privada, que são adquiridos no âmbito do mercado. Isso tudo influencia o modo de atendimento das mulheres no recorte da saúde reprodutiva.

No caso de Portugal, o crescimento do sistema suplementar propicia o aumento das taxas de cesariana no país para 32,9\%, sendo que o ideal seria por volta de 10\% ${ }^{11}$. Ainda assim, essa taxa se encontra em um patamar muito abaixo dos 55\% da apresentada no Brasil nesse período ${ }^{12}$ e, sobretudo, de algumas regiões do estado de São Paulo, como a região de São José do Rio Preto, que gira em torno de $80 \%$, de onde vieram as mulheres participantes deste estudo.

Dada a complexidade que envolve os processos migratórios e o impacto nas pessoas que os vivenciam, o objetivo deste estudo foi conhecer a percepção de imigrantes brasileiras sobre os cuidados de saúde reprodutiva oferecidos pelos serviços em Portugal.

\section{MÉTODO}

Trata-se de um estudo de abordagem qualitativa, realizado em 2016, com 13 mulheres brasileiras, em idade reprodutiva, provenientes da região de São José do Rio Preto, que moravam em Lisboa, Portugal.

Diante da dificuldade na identificação e localização dessas mulheres imigrantes em Portugal, foi utilizada a técnica' de amostragem em bola de neve ${ }^{13,14}$ a partir de suas famílias ${ }^{2}$, com as quais foi feito contato em São José de Rio Preto. Por esse processo, foram identificadas e contatadas, em Portugal, 21 mulheres, das quais 13 se disponibilizaram a participar da pesquisa e correspondiam aos critérios de inclusão (acesso ao SNS e saúde suplementar, estavam grávidas ou lá tiveram filhos).

Mediante agendamento prévio, foram realizadas entrevistas com essas mulheres em locais por elas indicados ${ }^{3}$, buscando estabelecer uma interação ${ }^{15}$ com elas e compreender como se dava a vivência da sua saúde reprodutiva no contexto dos serviços em Portugal.

A entrevista foi semiestruturada, com um roteiro que contemplou questões a respeito das dificuldades encontradas em Portugal, nos serviços de saúde reprodutiva, e possíveis preconceitos sofridos por elas. Todas as entrevistas foram gravadas e transcritas integralmente.

A análise das entrevistas foi realizada por meio da identificação de temas recorrentes nos discursos, buscando a explicitação dos conteúdos das diversas falas. O critério para o agrupamento dos temas foi a similaridade do seu significado ${ }^{16}$.

Em seguida, foi extraída uma história social, ou seja, uma realidade coletiva por meio de fatos singulares das mulheres que migraram da região de São José do Rio Preto para Portugal.

\section{RESULTADOS E DISCUSSÃO}

As 13 mulheres entrevistadas apresentavam algumas características semelhantes, como: a maioria estava na faixa de 30 a 40 anos, sendo apenas duas abaixo de 30 anos, e todas residiam há mais de cinco anos em Portugal. Em termos de escolaridade: cinco tinham o Ensino Fundamental incompleto, cinco cursavam o Ensino Médio e três possuíam formação superior; e a maioria trabalhava no setor de serviços. Nove tiveram filhos em Portugal, sendo seis no SNS e três no sistema suplementar.

Este estudo mostra que as brasileiras que moravam em Portugal, bem como a de outras nacionalidades, sofriam preconceito por serem imigrantes. Elas relataram ter sofrido algum grau de preconceito em Portugal por serem brasileiras: "[...] não em relação à lei propriamente dita",

\footnotetext{
A técnica de amostragem em bola de neve é indicada e utilizada quando é investigada uma população difícil de ser encontrada. A partir da identificação de um informante-chave, nomeado como semente, são localizadas outras informantes com o perfil para a pesquisa, e, a partir dessas outras indicações, sucessivamente, até não haver novos contatos ou não trazerem informações novas ao quadro de análise ${ }^{14}$.

2 A partir da identificação de uma família (informante-chave) em São José do Rio Preto que tinha um parente (filha, sobrinha, prima etc.) que havia migrado para Portugal, era solicitado o contato dele em Portugal e também indagado se conhecia outras famílias nessa situação e a possibilidade de fornecer o contato delas. Esse processo levou à identificação de 21 mulheres que haviam migrado para Portugal.

3 Geralmente, eram espaços públicos (praças, shopping, restaurante/café etc.) próximos aos locais de trabalho, em horários de almoço ou após o expediente.
} 
mas no que se refere às pessoas que as discriminavam e as julgavam, além dos funcionários que não informavam os direitos que elas tinham.

Há ainda uma acentuação dessa exclusão das imigrantes de origem brasileira, associada, frequentemente, a estereótipos de mulher com grande sensualidade ${ }^{5}$, o que, no contexto da moralidade portuguesa, significa que essas mulheres sofriam também com estereótipos de gênero e preconceitos de cunho sexista e sexual: "[...] a mulher brasileira é muito associada à prostituição"; "As brasileiras em Portugal são associadas ao sexo".

As expressões de preconceito normalmente se dão por meio de forte exclusão social do imigrante em situações do dia a dia, mas o grau da expressão desse preconceito depende de atributos individuais, tais como escolaridade, ocupação profissional e aparência física.

Para além do preconceito sentido da população local, há relatos de preconceito entre as próprias brasileiras:" [...] eu percebo que as brasileiras bem-sucedidas [mais estável, com emprego ou família] não querem mais contato com as brasileiras que não estão nessa situação, precisando de ajuda. Eu percebo isso". Geralmente, existe uma aproximação, um apoio dos imigrantes brasileiros residentes em Portugal, mas que se dá mais por ocasião da chegada ao país.

Segundo o estudo ${ }^{7}$ que aborda as redes sociais dos brasileiros em Portugal, o processo de chegada dos imigrantes é amenizado pelo apoio recebido da rede de amigos, conhecidos ou mesmo de algum familiar já estabelecido em Portugal, seja pela indicação de local para morar ou ainda pela indicação do primeiro emprego. Contudo, a relação estabelecida não se reveste de caráter de uma solidariedade, mas de uma empatia ética, ou seja, ela se dá mais no momento da chegada e, aos poucos, passa a ter uma descontinuidade dessa rede, sobretudo se for percebida uma possibilidade de concorrência entre os sujeitos.

\section{Acesso SNS x suplementar}

De uma forma geral, as entrevistadas relataram "desconforto" e "estranhamento" quando procuraram os serviços de saúde reprodutiva no SNS. Com relação ao parto, mencionaram sofrimento por causa da falta de analgesia e de pouca assistência dos profissionais. Além disso, contaram que eram os enfermeiros que conduziam o parto, que eles não faziam cesarianas e nem sempre utilizavam analgesia correta. "[...] as enfermeiras são, muitas vezes, as que fazem o parto, e o médico participa raramente"; "[...] no serviço público, não aplicam anestesia, eles falam que com a anestesia a mulher não colabora com o parto normal, mas no particular eles aplicam".

Os serviços de saúde particulares foram avaliados como melhores, quando utilizados por elas, do que os serviços do SNS. O acompanhamento pelo mesmo médico, a escolha do profissional e a discussão sobre o tipo de procedimento foram citados como fatores positivos nos serviços particulares, além da realização da cesariana. "[...] Quando fiquei grávida, fiquei apavorada, dizem que aqui [Portugal] não fazem cesariana, aí fui procurar médico no particular fui bem atendida e fiz cesariana", disse uma das entrevistadas.

A solicitação da cesariana pelas imigrantes brasileiras não deixa de ser a expressão da cultura de parte das brasileiras, que se inscreve na valorização das tecnologias de ponta, vinculada no estado de São Paulo, sobretudo com ênfase na região de São José do Rio Preto, de onde vieram essas mulheres. $O$ modelo de atenção obstétrica é altamente medicalizado, sendo o médico o único profissional capacitado para a assistência ao parto ${ }^{17}$. Acredita-se que esse modelo esteja interiorizado na maioria das mulheres desse estado, o que inclui as imigrantes entrevistadas; assim, a solicitação da cesariana por essas brasileiras expressa os efeitos da cultura da cesariana. Desse modo, compreender essa informação da origem das constantes solicitações de parto cirúrgico da parte das brasileiras seria fundamental para os profissionais da saúde de Portugal. Entender essa origem cultural facilitaria a orientação e os cuidados corretos das pacientes, o que, infelizmente, não acontece.

O crescimento dos convênios que atuam no mercado de saúde português favorece o parto cirúrgico, pois a prestação de serviços, neste caso, privilegia cuidados individuais e entende a mulher como consumidora do serviço de saúde, o que dilui sua condição de imigrante.

Segundo as entrevistadas, no entanto, em Portugal, "diferentemente do Brasil", não havia necessidade de se ter um plano de saúde privado, "sempre há disponibilidade para as consultas" 
no SNS, diferentemente do SUS no Brasil, onde consideravam como tendo acesso mais difícil. Contudo, afirmaram que, ao disporem de algum recurso financeiro, elas preferiam adquirir um plano privado. O motivo alegado pela maioria delas foi que, no privado, era possível escolher o médico, realizar cesariana, além de não sentirem o preconceito relacionado ao fato de serem brasileiras, o que as deixava mais seguras e confortáveis.

As solicitações constroem um eixo explicativo a ser mais bem explorado, atendendo não só à complexidade de sua consideração, mas também à possibilidade de fornecer elementos articuladores dos fatores que fragmentam a realidade complexa de uma mulher imigrante em terras estrangeiras.

De acordo com os relatos, as imigrantes brasileiras não se sentiam pertencentes à sociedade portuguesa. Afirmavam que havia um preconceito que permeava as relações e instituições, inclusive nos serviços de saúde. Para as entrevistadas, o preconceito era real, e alegavam que era reforçado pelos profissionais que as atendiam. “[...] o primeiro médico foi muito 'mal-educado.' Quando eu esquecia algum documento, ele dizia: 'só pode ser brasileira.' Consegui mudar de médico, e esse nunca maltratou, mas é de pouca conversa".

Há um consenso, tanto de quem utilizou os serviços de saúde públicos como os particulares, de que os médicos eram mais fechados. Segundo as entrevistadas, eles reclamavam que as brasileiras falavam muito, tendo opinião para todos os assuntos. Apontaram também que, quando ficavam grávidas, eram mais respeitadas pelo médico do que as que iam apenas para consulta ginecológica.

Essa percepção ilustra, de forma emblemática, o preconceito sofrido por elas, caracterizando certa "sacralidade" da maternidade, enquanto outras necessidades relacionadas à saúde reprodutiva, pode-se dizer, não são valorizadas por estarem associadas ao trabalho sexual, sensualidade e com as consequências que isso implica.

Com relação aos partos realizados no SNS, estes têm uma associação entre o fato de serem conduzidos por enfermeiros e a presença de dor, sofrimento e preconceito. De acordo com as falas das entrevistadas, esses eventos podiam ser evitados nos serviços suplementares, tendo em vista a possibilidade de se realizar a cesariana, o que representava uma solução para evitar os medos tanto do parto normal como da estigmatização socialmente produzida ${ }^{18,19}$

De acordo com os relatos das entrevistadas, foi observado que, apesar de o SNS incluir em seu princípio "a equidade", em que todos tinham direito aos cuidados de saúde reprodutiva, os protocolos estabelecidos sofriam interferência dos estereótipos de gênero e preconceitos com relação à escolha do tipo de parto e à condição de imigrante dessas mulheres. Além disso, foi percebido que havia um caráter econômico envolvido também, pois mulheres que podiam pagar por serviços privados recebiam melhores atendimentos.

\section{CONSIDERAÇÕESFINAIS}

Os tratamentos dispensados às imigrantes não são iguais aos das outras mulheres, pois envolvem um leque de relações que perpassam aspectos clínicos, na maioria das vezes condicionados por questões sociais e, mais diretamente, de financiamento dos procedimentos.

Há diferentes perfis de brasileiros em Portugal, que variam segundo o status socioeconômico, seus estados de origem, níveis de educação e experiências prévias com os sistemas de saúde. Isso faz com que as experiências desses imigrantes no sistema de saúde sejam diversas também em seu acesso.

Mesmo assim, há possibilidades de se levantarem algumas considerações, tais como o acesso à saúde ser permeado por preconceitos, dificultando a saúde universal.

A imigrante de origem brasileira frequentemente está associada aos estereótipos de uma mulher feminina com grande sexualidade, o que justifica que, quando ficam grávidas, são mais respeitadas pelos profissionais do que quando vão apenas para consulta ginecológica.

A solicitação da cesariana por elas não deixa de expressar os efeitos da cultura da cesariana, que se inscreve na valorização das tecnologias de ponta que trazem da região de onde vieram; mas também não se pode deixar de considerar a manifestação explícita da solicitação por parte das entrevistadas. 
Essas questões devem ser mais bem trabalhadas em futuros estudos, pois há necessidade de se verificar como fatores culturais, por exemplo, papéis sociais de gênero, hábitos tradicionais, linguagem e padrões de comunicação, podem condicionar a utilização dos serviços de saúde materna e cuidados pré-natais por parte das mulheres imigrantes.

\section{REFERÊNCIAS}

1. Morais MS, Lopes JC, Rocha LMV, Chiaravalloti RM, BrienzVMS. Retrato de uma região: impacto da indústria sucroalcooleira, indicadores de saúde e a percepção dos gestores. Saude Soc. 2018;27(3):922-8. http:// dx.doi.org/10.1590/s0104-12902018171658.

2. Masanet E, Padilla B, Ortiz A, Plasa S, Rodrigues E. Barreiras Socioeconómicas no acesso à saúde maternoinfantil das mulheres imigrantes na área metropolitana de Lisboa. In: Martins MIC, Marques AP, Costa NR, Matos A, organizadores. Trabalho em saúde, desigualdades e políticas públicas. Braga: Centro de Investigação em Ciências Sociais (CICS-UM), Universidade do Minho; Rio de Janeiro: Fiocruz; 2014. Cap. 1, p. 17-30.

3. Topa BJ, Neves S, Nogueira C. Imigração e saúde: (in) acessibilidade das mulheres imigrantes aos cuidados de saúde. Saude Soc. 2013;22(2):328-41. http://dx.doi.org/10.1590/S0104-12902013000200006.

4. Dias S, Rosário H. Saúde Sexual e Reprodutiva de Mulheres Imigrantes Africanas e Brasileiras: um estudo qualitativo [Internet]. Lisboa: ACIDI; 2009 [citado 19 Ago 2019]. Disponível em: http://bibliobase.sermais. pt:8008/BiblioNET/upload/PDF3/01922_OI_32.pdf

5. Padilla B, Plaza SH, Ortiz A. Avaliando as boas práticas em saúde e migração em Portugal: teoria, prática e política. Fórum Sociol. 2012;(22):33-41.

6. Brunelli, AF. Mais razão e menos emoção: o discurso de autoajuda para mulheres. Rev Estud Fem. 2018; 26(3):e49400.

7. Padilla B. Redes sociales de los brasileros recién llegados a portugal: ¿solidaridad étnica o empatía étnica? [Internet]. Revistas - Alternativas. Cuadernos de Trabajo Social. 2006;14:49-61 [citado 19 Ago 2019]. Disponível em: https://rua.ua.es/dspace/bitstream/10045/6505/1/ALT_14_04.pdf

8. França T, Padilla B. Imigração brasileira para Portugal: entre o surgimento e a construção mediática de uma nova vaga. Cad Estu Soc. 2018;33(2):1-30.

9. Fernandes D, Castro MCG. Migração e crise: o retorno dos imigrantes brasileiros em Portugal. Rev Inter Mob Hum. 2013;21(41):99-116. http://dx.doi.org/10.1590/S1980-85852013000200006.

10. Padilla $B$, Ortiz A. Fluxos migratórios em Portugal: do boom migratório à desaceleração no contexto de crise. Balanço e desafios. Rev Inter Mob Hum. 2012;20(39):159-84. http://dx.doi.org/10.1590/S198085852012000200009.

11. PORDATA - Bases de dados Portugal Contemporâneo. Partos nos hospitais oficiais: total e por tipo [Internet]. Lisboa: Fundação Francisco Manuel dos Santos; 2018 [citado 19 Ago 2019]. Disponível em: https://www. pordata.pt/Portugal/Partos+nos+hospitais+oficiais+total+e+por+tipo-1514

12. Brasil. Ministério da Saúde. Sistema de Informação sobre Nascidos Vivos - SINASC [Internet]. Brasília: Ministério da Saúde; 2014 [citado 15 Jun 2015]. Disponível em: http://tabnet.datasus.gov.br/cgi/tabcgi. exe?sinasc/cnv/nvSP.def

13. Bernand HR. Research methods in anthropology: qualitative and quantitative. 4th ed. Lanham (MD): Altamira Press; 2006.

14. Vinuto J. A amostragem em bola de neve na pesquisa qualitativa: um debate em aberto. Temáticas. 2014;22(44):203-20. http://dx.doi.org/10.20396/tematicas.v22i44.10977.

15. Fraser MTD, Gondim SMG. Da fala do outro ao texto negociado: discussões sobre a entrevista na pesquisa qualitativa. Paidéia. 2004;14(28):139-52. https://doi.org/10.1590/S0103-863X2004000200004.

16. Bardin L. Análise de conteúdo. Lisboa: Edições 70; 2009.

17. Patah LE, Malik AM. Models of childbirth care and cesarean rates in different countries. Rev Saude Publica. 2011 fev;45(1):185-94. http://dx.doi.org/10.1590/S0034-89102011000100021. PMid:21181056.

18. Capelli F, Rocha L, Zampieri MF, Gregorio VRP, Custodio ZAO. Fatores determinantes para a preferência da mulher pela cesariana. Texto Contexto Enferm. 2015;24(2):336-43.

19. Leal MC, Pereira APE, Domingues RMSM, Theme MM Fa, Dias MAB, Nakamura-Pereira M, et al. Intervenções obstétricas durante o trabalho de parto e parto em mulheres brasileiras de risco habitual. Cad Saude Publica. 2014;30(Supl):17-47. http://dx.doi.org/10.1590/0102-311X00151513. 